\title{
Parental age effects on the female fitness traits in Drosophila melanogaster
}

\author{
Fathemeh Tavakkoli ${ }^{1}$ and Abolhasan Rezaei $^{2}{ }^{*}$ \\ 1- Drosophila stock centre, Department of Zoology-Mysore University, India \\ 2- *Department of Genetics-School of Basic Science, Tonekabon Branch, Islamic \\ Azad University, Tonekabon, Iran. \\ a.rezaei@tonekaboniau.ac.ir
}

ABSTRACT

The female age including, 2-3 days aged old (young aged), 17-18 days aged old (middle aged) and 31-32 days aged (old aged) mated with virgin male (5-6 days aged old). The results by SPSS software analayzed that in almost all of experimental young aged old had significantly level than middle and old age. Fecundity elements, in young age was greater than middle and old age, also the female ovariole elements when mated with male young age was more than middle and old age. This variability suggests that female age effected on the male reproductive performance and female fitness traits. Therefore, the female age specific possibility occurrence on reproductive access as follows by: young $>$ middle $>$ old aged.

Keywords: D. melanogaster, female fitness traits, female ovariole, fecundity.

\section{INTRODUCTION}

In the species of Drosophila, aging is well known that affecting many life history traits such as viability and reproduction performance (Moore and Moore 2001). In Drosophila melanogaster, an extreme study has discussed effect of aging on the reproductive performance (Luckinbill et al. 1984; Rose 1984; Partridge and Fowler 1992; Zwaan et al., 1995; Partridge and Barton, 1996). Furthermore, female age effect is important on the male mating success and among of female fitness traits such as fecundity, fertility, courtship activities and longevity in Drosophila melanogaster (Anderson et al., 1973; Brittnacher, 1981). Fecundity and ovariole counting as a fitness component can be affected by the presence of males and females (Nuzhdin et al. 1997; Leips and Mackay, 2000). Hansen and Price 1995; Brooks and Kemp 2001, proposed, that age is often regarded as an important factor for mate choice. The aging cause of decreasing or increasing viability and fertility might be contributed to a negative relationship between age and mate quality. In Drosophila melanogaster sexual selection has played an integral role in the patterns and fitness consequences of female mate choice (Spieth 1952; Partridge 1980; Fowler \&Chapman et al. 1995; Gromko \& Markow 1993). Kokko et al, 2003, also shows the role of sexual behavior in affecting patterns of mate choice, since the role that parental age effect plays in the genetic basis of aging has been largely neglected (Nuzhdin et al. 1997; Leips and Mackay, 2000). In this study we addressed the issues associated with age-based and female ovariole elements. Firstly, we assessed whether, when presented with a choice of young, middle-aged, and old females, with fecundity and longevity elements. Secondly, we performed a female age on the female reproductive performance, such as female mature ovariole and female immature ovariole with a constant male age in Drosophila melanogaster. 


\section{MATERIALS AND METHODS}

a) Selection of female age-classes: The isofemale lines of Drosophila melanogaster collected from the national Drosophila stock center ,department of Zoology(UOM)India. When adults emerged, virgin females and unmated males were isolated within 3 $\mathrm{h}$ of eclosion and were aspirated into a new vial containing wheat cream agar medium. These flies were aged as required for the experiment. In order to provide uniform environmental conditions for females of different age classes, female flies collected first were assigned to 32-33 d (older); females flies collected next were assigned to 17-18 d (middle-aged). Following this, female flies collected were assigned to 2-3 d (younger).

b) The influence of female age on the number of mature and immature ovarioles: Unmated males and virgin females were isolated during the late pupal stage and maintained in the above lab conditions in separate vials. To count the number of ovarioles, virgin young, middle-age and old female flies were collected. The ovary consists of left and right lobe. In each of the lobe both matured and immatured ovarioles will be present which can be easily demarcated by the completely or halffilled lobes of the ovarioles. Females of all three age class were used to perform the experiment. Each of the female flies was dissected in physiological saline under the stereomicroscope. The left and right ovaries were dissected out and the number of ovariolees in each lobes were calculated respectively also the number of mature and immature ovarioles were calculated for the same. 50 trials were separately conducted for each of the 3 female age classes. One-way ANOVA was carried out using SPSS (Version 10.1).

\section{RESULTS}

The female fecundity: The female egg classes (young, middle and old) mated with 56 days male age. The success mated female were separated and kept in the new vial contain wheat cream medium agar. Female egg laid every day were counted and recorded. Result of mean value between three female age classes shows, young female age more than egg laid middle and old female age. In figure 1, shows that the length of graph in young female longer than middle and old age female.

Longevity experiment: In the present study were used three female age classes, (young, middle and old) for longitudinal studies. In related to young female were mated with 5-6 days aged male, after finished egg laid female were kept in the vial contain wheat cream medium agar until dead. The mean value of result between females are shown, young female age more than middle and old age female, In figure 1 , also are shown, longer of graph in young female longer than middle and old age female.

Female ovariole experiment: Mated younger female, middle-aged and older-female were individualy sacrified to count the number of ovarioles including, left mature and immature ovary, total left mature and immature ovary, right mature and immature ovary and total right mature and immature ovary. The count number of ovarioles, each female was dissected in a drop of physiological saline using a binuclear stereomicroscope. Ovarioles of left mature and immature ovary were separated from one another with the help of fine needles. The number of ovarioles in each female was counted. Similarity that ovarioles of right mature and immature ovary were separated and was recorded. In figure 2 and 3, the mean ovariole of left mature and immature 
also total left mature and immature in old female age and young female age was more than young and middle and old and middle respectively. The number of ovarioles in right mature and immature, total right mature in old female and young female more than other female ages (Figure 4). Regarding number of total ovariole, young female age more than middle and old age female (Figure 5).

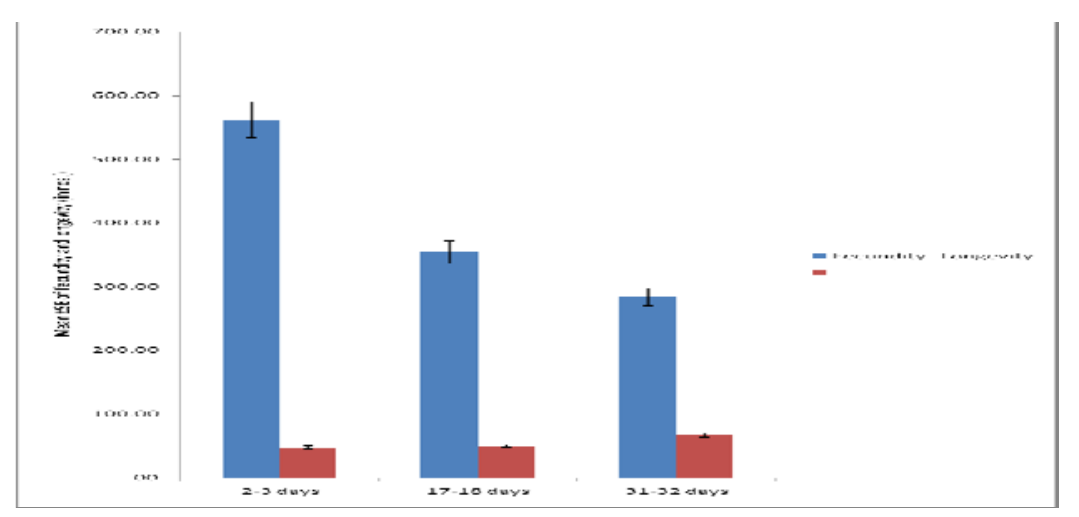

Fig. 1: The result of female fecundity and longevity studies between three female age classes (young, middle and old).

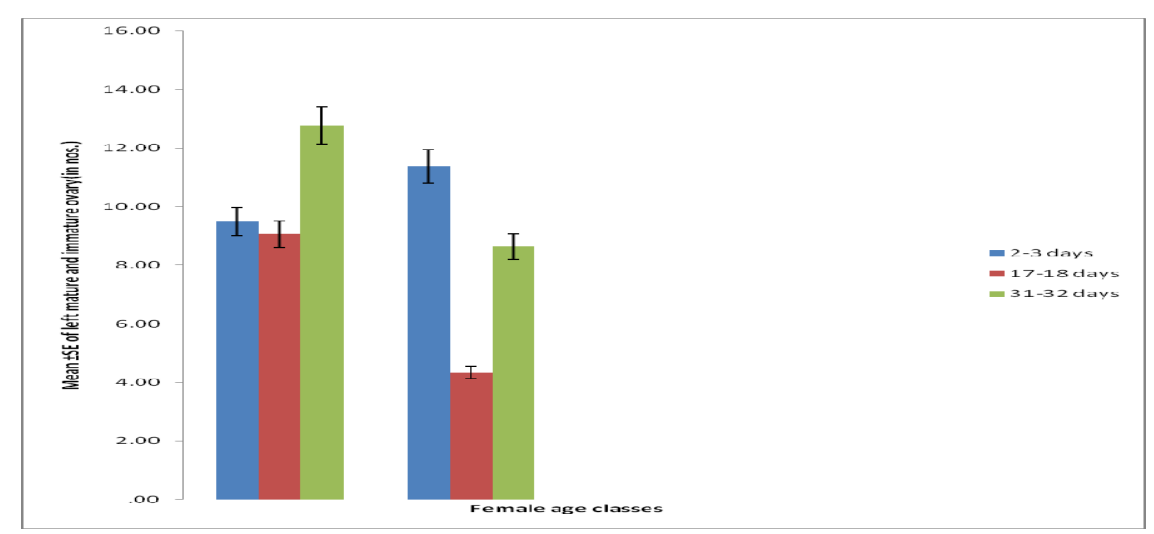

Fig. 2: Left mature and immature ovary between female age classes.(young, middle,old).

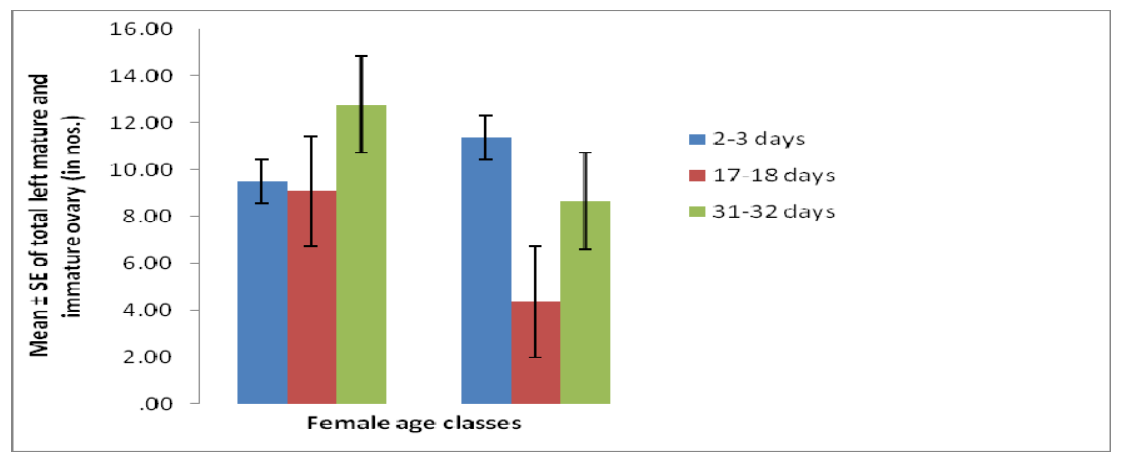

Fig. 3: Total left mature and immature ovary between female age classes.(young, middle,old). 


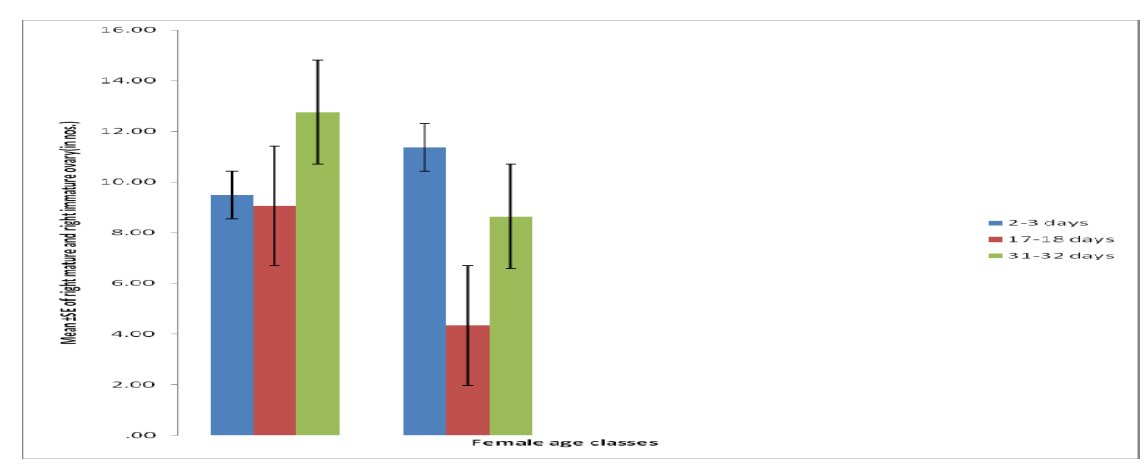

Fig. 4: Right mature and immature ovary between female age classes. (young, middle,old).

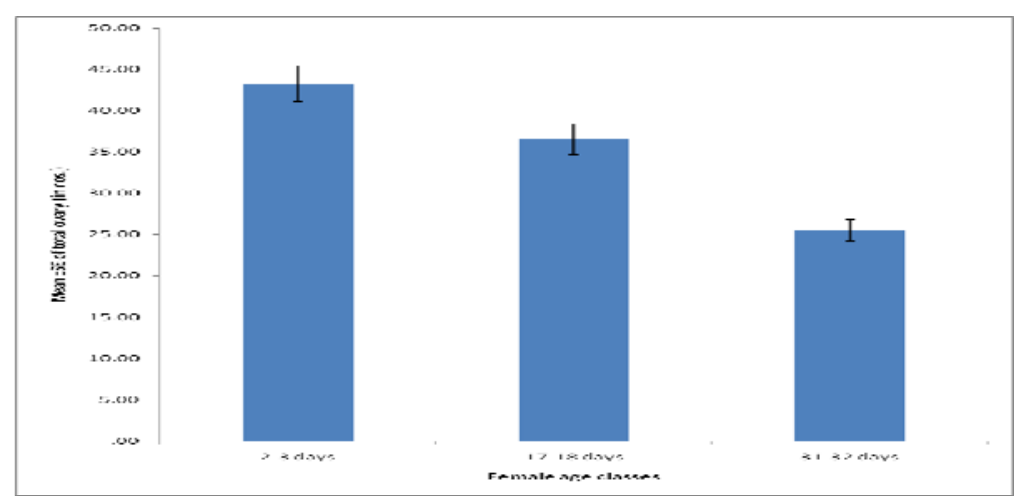

Fig. 5: Total ovary between female age classes.(young, middle,old).

\section{DISCUSSIONS}

Studies on the D. melanogaster reveals that males prefer to mate with middleaged females more frequently than younger and older females, suggesting that males of $D$. melanogaster exercise mate choice to obtain direct benefits in the form of greater fecundity. Thus, it appears that female age is an important determinant of male mate choice. In other words, males of $D$. melanogaster do not show the same levels of interest in females of different age classes. This confirms earlier studies of the existence of male mate choice for females in other insects (Bonduriansky 2001). Figure 1, shows that middle-aged females had significantly greater fecundity compared to younger and older females, suggesting that females of the same species show variations in reproductive potential across age. This confirms earlier work suggesting that female age is also one of the traits known to influence female fecundity and female ovariole elements, that of Bonduriansky (2001), in working on the evolution of male mate choice in insects who also found an influence of female age on female fecundity and female ovariols elements. Since the number of ovarioles is positively correlated with fecundity, we sacrificed females of different age classes to study variations in the number of ovarioles if any at different female ages. Figure 2 shows that middle-aged females had a significantly greater number of ovarioles than younger an older females, and follows a pattern of fecundity across female age. This again confirms earlier studies of the influence of female age on reproductive success in other insects (Bonduriansky 2001). From our results, it was also noted that in $D$. melanogaster, middle-aged females had a significantly greater number of ovarioles and higher fecundity compared to younger and older females. Therefore, it is advantageous for males to mate with middle-aged females compared to younger and 
older females. However, it is not known why older female had fewer ovarioles than younger and middle-aged females. We do not know whether they reabsorb them as the females age. Studies in insects also found a positive correlation between female size and the number of ovarioles (Avent et al. 2008). Even in Drosophila, studies of Avent et al. 2008, also found a positive correlation between female size and the number of ovarioles. Therefore, in the present study, flies which were sacrificed to study the ovariole number were also used to measure wing length to understand relationships among female age, wing length, and ovariole number. It was found that there was an insignificant difference in the mean female wing length among females of different age classes. This suggests that female age has no influence on female body size, but has a significant influence on ovariole number. These studies suggest that females of $D$. melanogaster differ in reproductive potential, i.e., fecundity and ovariole number across different female age classes, but they did not significantly differ in body size across the different female age classes.

\section{ACKNOWLEDGMENTS}

This work had financially supported by the research council of Islamic Azad University Tonekabon branch, Iran.

\section{REFERENCES}

Anderson, W.W. (1973). Genetic divergence in body size among experimental populations of Drosophila pseudoobscura kept at different temperatures. Evolution, 27: 278-284

Avent, T. D.; Price Tar and Wedell, N. (2008). Age based female preference in the fruit Fly D. pseudoobscura. Animal Behaviour., 75 (4): 1413-1421.

Bonduriansky, R. (2001). The evolution of male mate choice in insects: a synthesis of ideas and evidence. Biological Reviews., 76:305-339.

Brittnacher, J.G. (1981). Genetic variation and genetic load due to the male reproductive component of fitness in Drosophila. Genetic., 97:719-730.

Brooks, R. and Kemp, D.J. (2001). Can older males deliver the good genes?. Trends in Ecology and Evolution., 16:308-313.

Chapman, T.; Liddle, L.F.; Kalb, J.M.; Wolfner, M.F. and Partridge, L. (1995). Cost of mating in Drosophila melanogaster females is mediated by male accessory gland products. Nature., 373:241-244.

Dickinson, J. L. (2001). Extrapair copulations in western bluebirds (Salia mexicana): female receptivity favors older males. Behav. Ecol. Sociobiol., 50:423-429.

Grant, B.R. and Grant, P. R. (1987). Mate choice in Darwin's finches. Biol. J. Lin. Sot., 32:247-270.

Gromko, M.H. and Markow, T.A. (1993). Courtship and remating in field populations of Drosophila. Animal Behavior., 45:253-26.

Gross, R. and Nilsson, J. (1995). Application of heteroduplex analysis for detecting variation within the growth hormone 2 gene in Salmo trutta L. brown trout. Heredity., 74:286-295.

Hansen, T.F. and Price, D. K. (1995). Good genes and old age, do old mates provide superior genes? Evol. Biol. (8)759-778.

Kern., A. D.; Jones, C. D. and Begun, D. J. (2004). Molecular population genetics of male accessory gland proteins in the Drosophila simulans complex. Genetics., 167:725-735. 
Kokko, H. (1997). Evolutionary stable strategies of agedependent sexual advertisement. Behav. Ecol. Sociobiol., 41:99-107.

Leips, J. and Mackay, T.F.C. (2000). Quantitative trait loci for life span in Drosophila melanogaster: interactions with genetic back-ground and larval density. Genetics., 155:1773-1788.

Lubkinbill, L.R.; Arking, M.J.; Clare, W.C. and Cirocco, S. (1984). Buck. Selection of delayed senescence in Drosophila melanogaster. Evolution., 38:996-1003.

Moore, P.J. and Moore, A. J. (2001). Reproductive aging and mating: the ticking of the biological clock in female cockroaches. Proc Natl Acad Sci USA., 98: 9171-9176.

Partridge, L. (1980). Mate choice increases a component of offspring fitness in fruit flies. Nature, 283: 290-291.

Partridge, L. and Barton, N.H. (1996). On measuring the rate of ageing. Proceedings of the Royal Society of London: Series B, 263: 1365-1371.

Partridge, L. and Fowler, K. (1992). "Direct and correlated responses to selection on age at reproduction in Drosophila melanogaster." Evolution., 46: 76-91.

Rose, M.R. (1984). Laboratory evolution of postponed senescence in Drosophila melunoguster. Evolution., 38:1004-1010.

S. V., Nuzhdin., E. G.; Pasyukova., C.L.; Dilda., Z.B.; Zeng, T. F. C. and Mackay, (1997). Sex-specific quantitative trait loci affecting longevity in Drosophila melanogaster. Proc. Natl. Acad. Sci. U.S.A., 94:9734-9739.

Spieth, H.T. (1952). Mating behavior within the genus Drosophila(Diptera). Bulletin American Museum National History, 99:395-474.

SPSS, I. (1999). SPSS for Windows, 10.0.1. SPSS.

Zwaan, B.; Bijlsma, R. and Hoekstra, R. E. (1995). Direct selection on life span in Drosophila melanogaster. Evolution., 49:649-659. 